\title{
Anomalous heat transport in binary hard-sphere gases
}

\author{
Craig Moir, ${ }^{1,2}$ Leo Lue, ${ }^{3}$ Julian D. Gale,${ }^{2}$ Paolo Raiteri, ${ }^{2}$ and Marcus N. Bannerman ${ }^{1, *}$ \\ ${ }^{1}$ School of Engineering, University of Aberdeen, \\ Aberdeen, AB24 3UE, United Kingdom \\ ${ }^{2}$ Curtin Institute for Computation, Curtin University, \\ PO Box U1987, Perth, WA 6845, Australia \\ ${ }^{3}$ Department of Chemical and Process Engineering, \\ University of Strathclyde, James Weir Building, \\ 75 Montrose Street, Glasgow G1 1XJ, United Kingdom
}

(Dated: March 3, 2019)

\begin{abstract}
Equilibrium and non-equilibrium molecular dynamics (MD) are used to investigate the thermal conductivity of binary hard-sphere fluids. It is found that the thermal conductivity of a mixture can not only lie outside the series and parallel bounds set by their pure component values, but can lie beyond even the pure component fluid values. The MD simulations verify that revised Enskog theory can accurately predict non-equilibrium thermal conductivities at low densities and this theory is applied to explore the model parameter space. Only certain mass and size ratios are found to exhibit conductivity enhancements above the parallel bounds and dehancement below the series bounds. The anomalous dehancement is experimentally accessible in helium-hydrogen gas mixtures and a review of the literature confirms the existance of mixture thermal conductivity below the series bound and even below the pure fluid values, in accordance with the predictions of revised Enskog theory. The results reported here may reignite the debate in the nanofluid literature on the possible existence of anomalous thermal conductivities outside the series/parallel bounds as this work demonstrates they are a fundamental feature of even simple fluids.
\end{abstract}


There has been a great deal of interest in increasing the transport rate of heat through fluids by the addition of nanometer-sized solid particles. Initial experiments on these nanosuspensions demonstrated significant enhancements of the thermal conductivity [1]; however, later measurements on other mixtures generated substantial controversy [2], with confusion over what results might be "unsurprising" and what might be deemed "anomalous". Disagreements in reported values led to a comprehensive benchmark study with double blind tests conducted between several institutions [3]. The primary conclusion was that the vast majority of the reported "enhancement" effects lie within the classical/continuum bounds given by the series $\lambda^{\perp}$ and parallel $\lambda^{\|}$limits of the thermal conductivity [4], which for a binary mixture are,

$$
\begin{aligned}
\frac{1}{\lambda^{\perp}} & =\frac{1-\phi_{2}}{\lambda_{1}}+\frac{\phi_{2}}{\lambda_{2}} \\
\lambda^{\|} & =\left(1-\phi_{2}\right) \lambda_{1}+\phi_{2} \lambda_{2},
\end{aligned}
$$

where $\phi_{2}$ is the volume fraction of component 2 in the mixture, and $\lambda_{1}$ and $\lambda_{2}$ are the fluid thermal conductivities for pure 1 and pure 2, respectively.

A small number of experimental results still remain outside these conventional bounds, such as the reported dehancements below the series limit for fullerene-water suspensions [5]. Hence, the question still remains; are results outside these bounds correct and, if so, what are the underlying mechanisms? Several physical mechanisms have been proposed in an effort to rationalize the behavior of these systems [4]; however, there is as-yet no unifying framework for predicting/explaining the thermal performance of nanofluid mixtures. To be able to understand these results, a deeper understanding of thermal conductivity and its underlying molecular mechanisms is required.

Some of the confusion in interpreting thermal conductivity arises from the different manners in which it can be defined. The most natural macroscopic/experimental definition arises from applying a temperature gradient, $\nabla T$, across a system and measuring the resultant heat flux, $\mathbf{J}_{q}$. The "observed" non-equilibrium thermal conductivity $\lambda_{N}$ is then defined through the following expression,

$$
\left\langle\mathbf{J}_{q}\right\rangle=-\lambda_{N} \nabla T
$$

where the brackets $\langle\cdots\rangle$ indicate the implicit averaging over time and volume this approach entails. 
In contrast, when considering hydrodynamic models it is natural to decompose the heat flux, $\mathbf{J}_{q}$, into contributions from thermal and mass diffusion of the individual species; however, this separation of these two effects is not unique and an arbitrary number of definitions of the thermal conductivity can be generated. The so-called mainstream, prime, and double prime definitions [6, 7] are the most common choices. The mainstream definition is used here as it is convenient for molecular dynamics simulations and is given below for binary systems,

$$
\mathbf{J}_{q}=-T^{-1} L_{u u} \nabla T-L_{u 1} T \nabla \frac{\left(\mu_{1}-\mu_{2}\right)}{T}
$$

where $L_{u u}$ is the mainstream thermal conductivity, $L_{u 1}$ is the mainstream thermal diffusivity of species 1 , and $\mu_{1}$ is the chemical potential of species 1 . One possible resolution to the ambiguity in the definition of $L_{u u}$ is to assume local steady-state conditions (zero mass flux) [4, 8] to yield the following expression,

$$
\mathbf{J}_{q}=-T^{-1}\left(L_{u u}-L_{u 1}^{2} L_{11}^{-1}\right) \nabla T=-\lambda \nabla T
$$

where $L_{11}$ is the mutual diffusion coefficient of species 1 through itself and the final equality implicitly defines the "steady-state" thermal conductivity, $\lambda$. The value of $\lambda$ is independent of the choice of mainstream, prime, or double prime fluxes; however, it is still distinct from $\lambda_{N}$ as it is not averaged over the non-equilibrium conditions of a system undergoing conduction but is instead evaluated at a single temperature, concentration, and density [29]. The first hurdle of this paper is to establish that the macroscopic $\left(\lambda_{N}\right)$ and microscopic $(\lambda)$ definitions of the thermal conductivity are equivalent at steady state.

In principle, atomistic non-equilibrium molecular dynamics (NEMD) simulations can be used to directly measure the heat flux $\mathbf{J}_{q}$ through a system's boundaries to obtain the "observed" non-equilibrium thermal conductivity, $\lambda_{N}$. Equilibrium simulations can then be used to measure $\lambda$ for comparison, as well as to elucidate any underlying mechanisms behind "anomalous" behavior. Unfortunately, large and long-duration non-equilibrium simulations are required to directly study heat conduction in nanofluids [9], and so only a limited range of molecular models and techniques are computationally accessible using this method. Due to these limitations, previous simulation work has primarily focused on equilibrium simulations of nanofluids at fixed size and mass asymmetries between the fluid molecule and nanoparticle [7, 10 14] with only a few studies at larger asymmetries (e.g., [8, 15]). As 
equilibrium molecular simulations are conducted at a single temperature and concentration, Eq. (3) cannot be used, thus the equivalence between $\lambda_{N}$ and $\lambda$ cannot be conclusively established.

In this work, NEMD simulations of binary hard-sphere mixtures, consisting of spheres of diameter $\sigma_{1}$ and mass $m_{1}$ and spheres of diameter $\sigma_{2}$ and mass $m_{2}$, confined between two smooth parallel walls (see Fig. 1) are performed using the DynamO [16] event-driven molecular simulation package. The hard sphere model is both computationally accessible and well-described by revised Enskog theory [7, 17, 18] which can be used to yield accurate predictions of $\lambda$ at low densities [15]. Although the hard-sphere model is simple, it qualitatively captures the fundamental effects of density, molecular size, and mass on the transport coefficients in gases. This paper explores conditions close to the ideal gas limit for simplicity (and to avoid crystallisation as $\sigma_{2} / \sigma_{1}$ becomes large); however, the results obtained in this limit are fundamental to the behavior of all fluids and comparision against experiments on gas mixtures can be made. This limit is also particularly interesting as the current discussion in nanofluids echoes previous controversy over reported dehancements in the thermal conductivity of $\mathrm{He}-\mathrm{H}_{2}$ gas mixtures [19]. Although the source of the original controversy (a sharp minimum in conductivity with concentration) was later shown to be unrepeatable [20, 21], a shallower minimum still remains and demonstrates that thermal conductivity can lie outside the series-parallel bounds and even beyond the pure fluid values. If this is correct, then it implies that such minima are also possible for nanofluid systems which are the subject of some controversy even today.

The first aim of this work is to establish an equivalence of the observed, $\lambda_{N}$, and steady-state, $\lambda$, thermal conductivities. This is conducted using a hard-sphere mass ratio of $m_{2} / m_{1}=2$ corresponding to a $\mathrm{He}-\mathrm{H}_{2}$ mixture with the approximate size ratio $\sigma_{2} / \sigma_{1}=260 / 289$, obtained from diffusion measurements [23]. A constant reduced pressure of $p \sigma_{1}^{3} /\left(k_{B} T\right)=0.01$ (where $k_{B}$ is the Boltzmann constant) is used and is set by adjusting the system density. This reduced pressure value corresponds to a packing fraction of approximately 0.005 over the studied mol fraction, $x_{2}$. Once the equivalence of $\lambda$ and $\lambda_{N}$ is confirmed in this system, a systematic exploration for "anomalous" thermal conductivities is carried out over the mass and size ratio parameter space using kinetic theory.

Parallel smooth walls are located at both ends of the simulation domain as illustrated in Fig. 1 with periodic conditions on all other boundaries. On collision with the wall, the nor- 


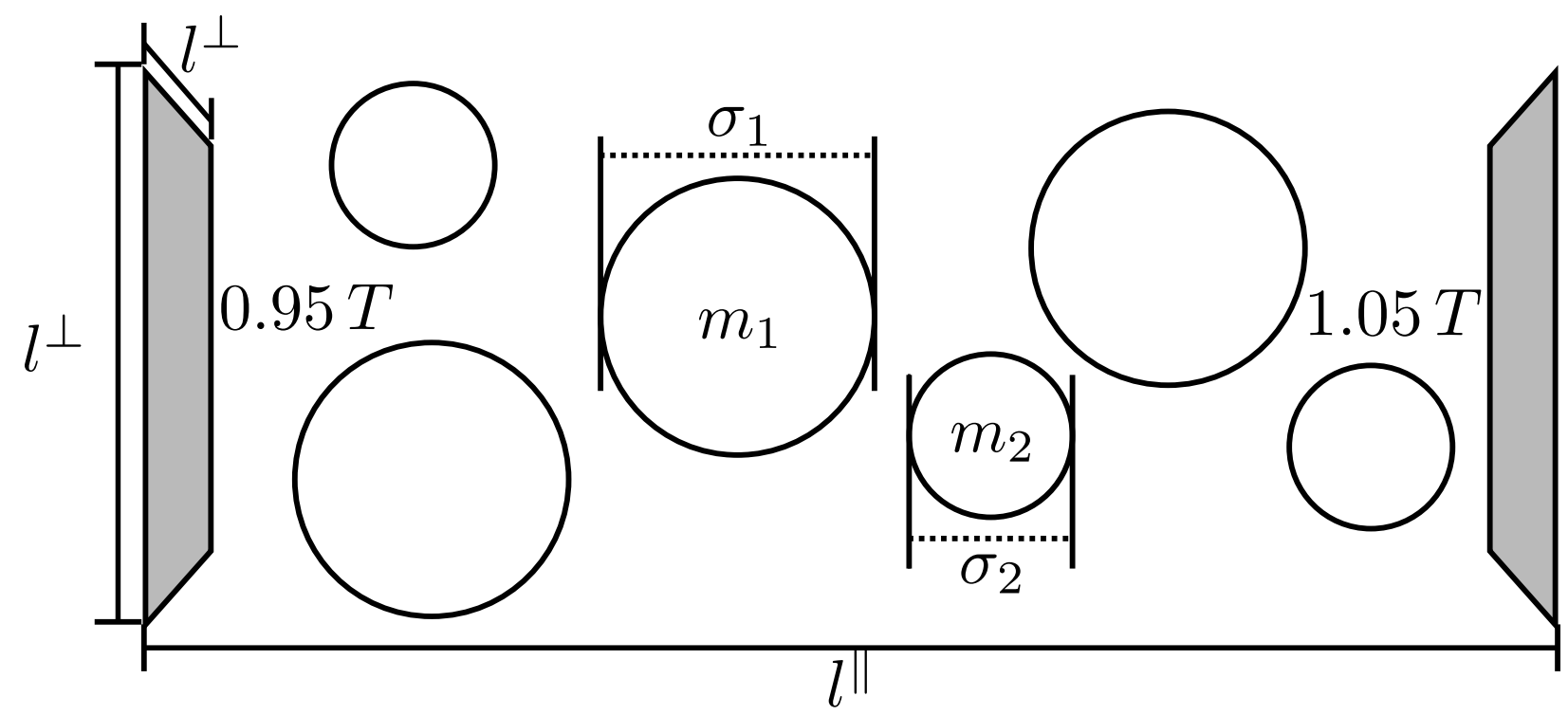

FIG. 1: A schematic of the system configuration used for the simulations reported here which use a hard-sphere model consisting of two species with diameters $\sigma_{1}$ and $\sigma_{2}$, and masses $m_{1}$ and $m_{2}$ respectively. For NEMD simulations, two walls are inserted into the simulation and a $10 \%$ temperature gradient is imposed via velocity reassignment on collision with the wall [22]. The total number of spheres $N$ and aspect ratio $l^{\|} / l^{\perp}$ of the simulation are varied to explore the effects of system size at a constant density/pressure.

mal component of a sphere's velocity is reassigned to a Maxwell-Boltzmann distribution [22] at a defined temperature. The heat flux is then measured through the following expression, $\left\langle\mathbf{J}_{q}\right\rangle=\langle Q\rangle l^{\|} /\left(l^{\perp}\right)^{2}$, where $\langle Q\rangle$ is the time-averaged rate of energy transferred to the simulation during sphere impacts with the walls averaged over both walls. The two walls have different temperatures set to $95 \%$ and $105 \%$ of the system temperature $T$. This value is a trade-off between inducing a sufficently large heat flux (compared to thermal fluctuations) and inducing inhomogeneity in the system.

An example of the inhomogeneity induced by the heat flux is given in Fig. 2, The temperature, number density, and concentration plots appear approximately linear. It is clear to see the effects of thermophoresis in the concentration profiles. These inhomogeneities make equating $\lambda_{N}$ and $\lambda$ suspicious as $\lambda$ is only evaluated at a single representative concentration, temperature, and density, whereas NEMD simulations measure an average thermal conductivity across the system as temperature, density, and concentration varies. Boundary 


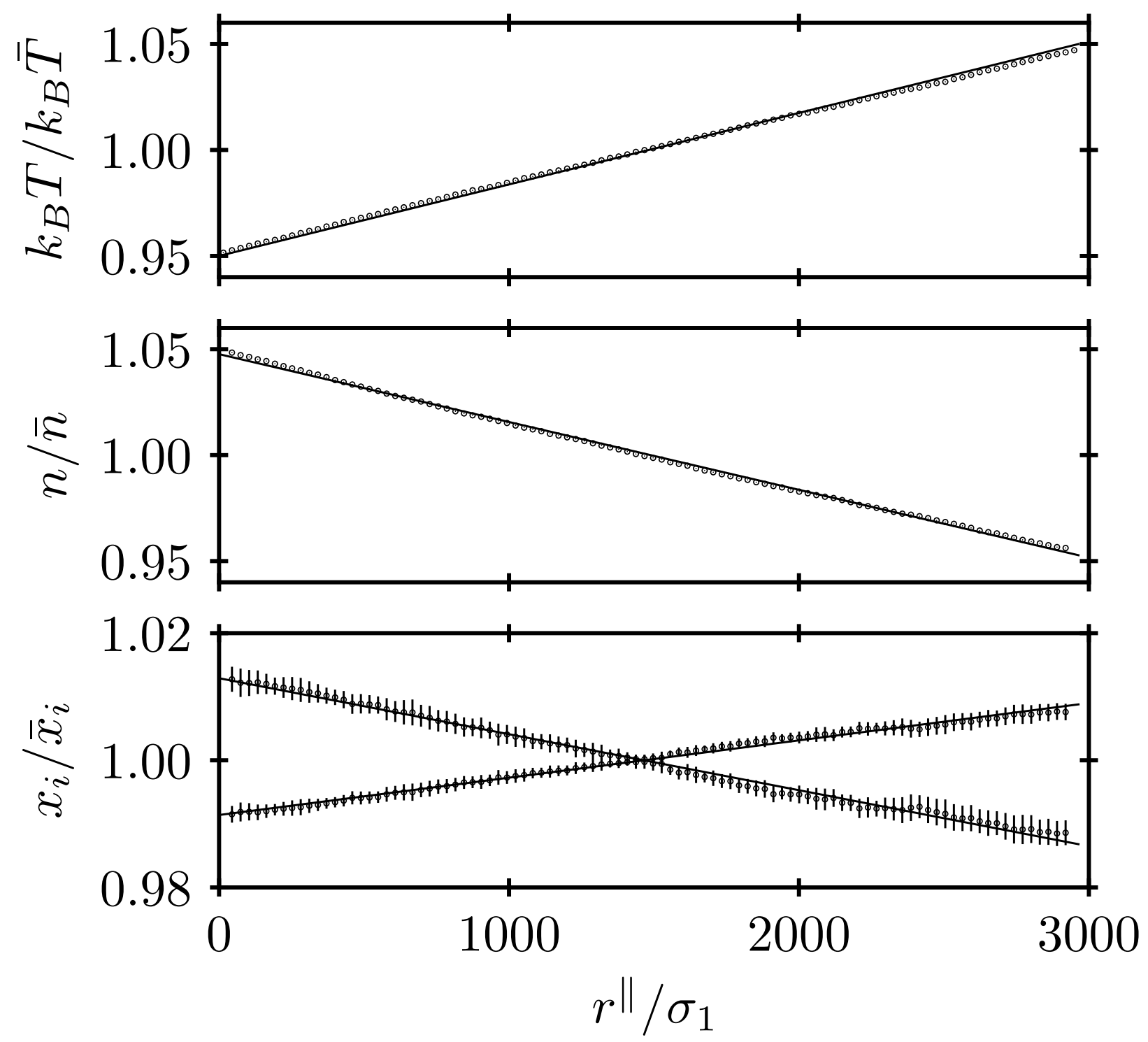

FIG. 2: The profiles of temperature, $k_{B} T$, number density, $n$, and species mol fraction, $x_{i}$, as a function of distance between the two heated walls, $r \|$ for a single representative simulation. This system has a mass ratio of $m_{2} / m_{1}=2$, a size ratio of $\sigma_{2} / \sigma_{1}=0.899654, N=102400$ spheres, and an aspect ratio of $l^{\|} / l^{\perp}=50$. All values are reduced by the average values for the system, which are $k_{B} \bar{T}=1, \bar{n} \approx p \sigma_{1}^{3} /\left(k_{B} T\right)=0.01$, and $\left(1-\bar{x}_{1}\right)=\bar{x}_{2}=0.6$. Error bars represent the standard deviation across 10 simulations and are smaller than the marker size for the temperature and number density fields. Solid lines are linear fits provided as a guide to the eye. 
layers with high thermal resistance also exist very close to the heated walls and induce a significant system-size dependence in the NEMD results.

To explore this system-size dependence, the wall temperatures are held fixed while the aspect ratio $l^{\|} / l^{\perp}$ and system size are varied with the number of spheres ranging from $N=5000$ up to $N=102400$. Each simulation is initialized in an FCC crystal lattice and equilibrated for $1000 \mathrm{~N}$ collisions before a further production run of $10000 \mathrm{~N}$ collisions to collect data. This procedure is repeated ten times at each state point and average values between the production runs are collected while the errors of the average measurements are estimated using the standard deviation of values between each production run. Results are reported here in reduced units, with $\sigma_{1}$ the unit of length, $m_{1}$ the unit of mass, and $k_{B} T$ the unit of energy.

A system-size dependence calculation for the mole fraction, $x_{2}=0.8$, in the $\mathrm{He}_{2} \mathrm{H}_{2}$ system is reported in Fig. 3. The figure demonstrates that the aspect ratio of a system is relatively unimportant and that the system length in the direction of conduction, $l^{\|}$, dominates the system-size effects (due to the boundary layer resistance near the walls). To estimate the infinite system-size (near-zero thermal gradient) value of the thermal conductivity, linear extrapolation is applied to systems with the three largest aspect ratios $\left(l^{\|} / l^{\perp}=10,25\right.$, and 50). This procedure yields a lower-bound for the thermal conductivity as the gradient of the system-size dependence monotonically increases with system size. The extrapolated $\lambda_{N}$ value is in excellent agreement with the equilibrium thermal conductivity $\lambda$ from revised Enskog theory evaluated at the average conditions of the system.

The above procedure is repeated over a range of mole fractions and the final infinitesystem extrapolated results are reported in Fig. 4. This system exhibits thermal conductivities that are far outside the predictions of classical approaches such as Maxwell theory or the limits of series and parallel resistance. In accordance with the experimental results for $\mathrm{He}_{2} \mathrm{H}_{2}$ [19 21], the system displays a minimum in the thermal conductivity below both the pure fluid thermal conductivities. This conclusively demonstrates that "anomalous" thermal conductivities are not only possible but a fundamental feature of simple molecular fluids such as the binary hard-sphere gas.

To further validate the Enskog and NEMD results, equilibrium simulations with $N=$ 32000 spheres in a cubic system are equilibrated for $1000 N$ events before being run for a further $100000 N$ events to calculate $L_{u u}, L_{u 1}$, and $L_{11}$ for this system. The Einstein form of 


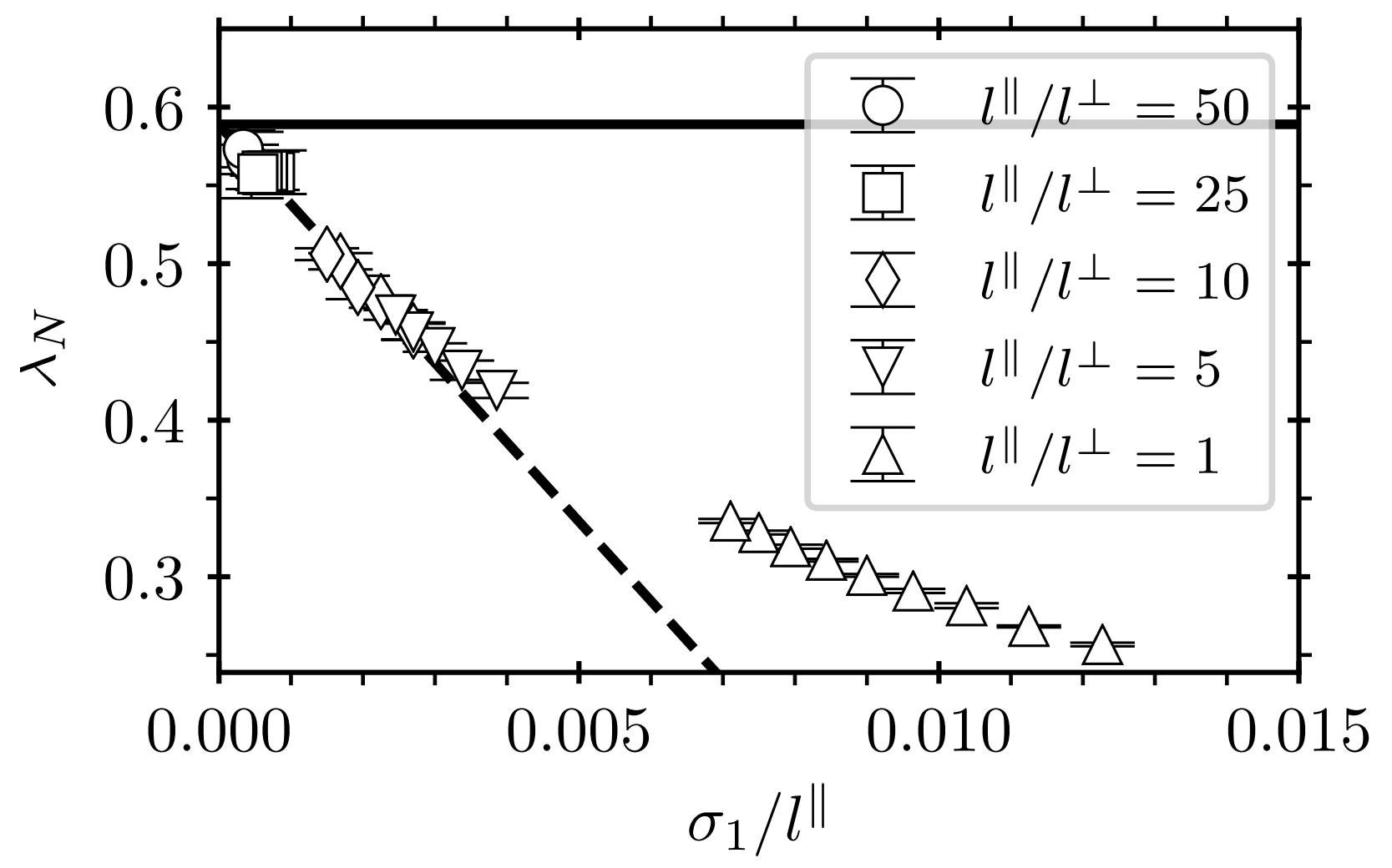

FIG. 3: The observed thermal conductivity $\lambda_{N}$ obtained from NEMD simulations as a function of the distance between the walls $\sigma_{1} / l^{\|}$from binary hard-sphere systems with varying numbers of spheres and aspect ratios at a mole fraction of $x_{2}=0.8$ for a $m_{2} / m_{1}=2, \sigma_{2} / \sigma_{1}=0.899654$, and $p \sigma_{1}^{3} /\left(k_{B} T\right)=0.01$. A linear fit to the data points obtained using an aspect ratio of 10,25 , and 50 (dashed line) is used to extrapolate to infinite system size and approaches the value of $\lambda$ predicted by revised Enskog theory (solid line).

the Green-Kubo relations [24, 25] is used with the first 15 mean free times of the correlation discarded to avoid ballistic motion and a maximum correlation time of 50 mean free times used to avoid correlations from the periodic boundary conditions. This is beyond the sound wave traversal time of the system; however, as the density is so low, correlations from the boundary conditions take much longer to establish than in higher density systems. The excellent agreement in Fig. 4 between revised Enskog theory and the equilibrium molecular dynamics completes the verification of the anomalous dehancement reported and the use of revised Enskog theory to further study the system at low densities.

The full parameter space of the binary hard sphere model is explored using revised En- 


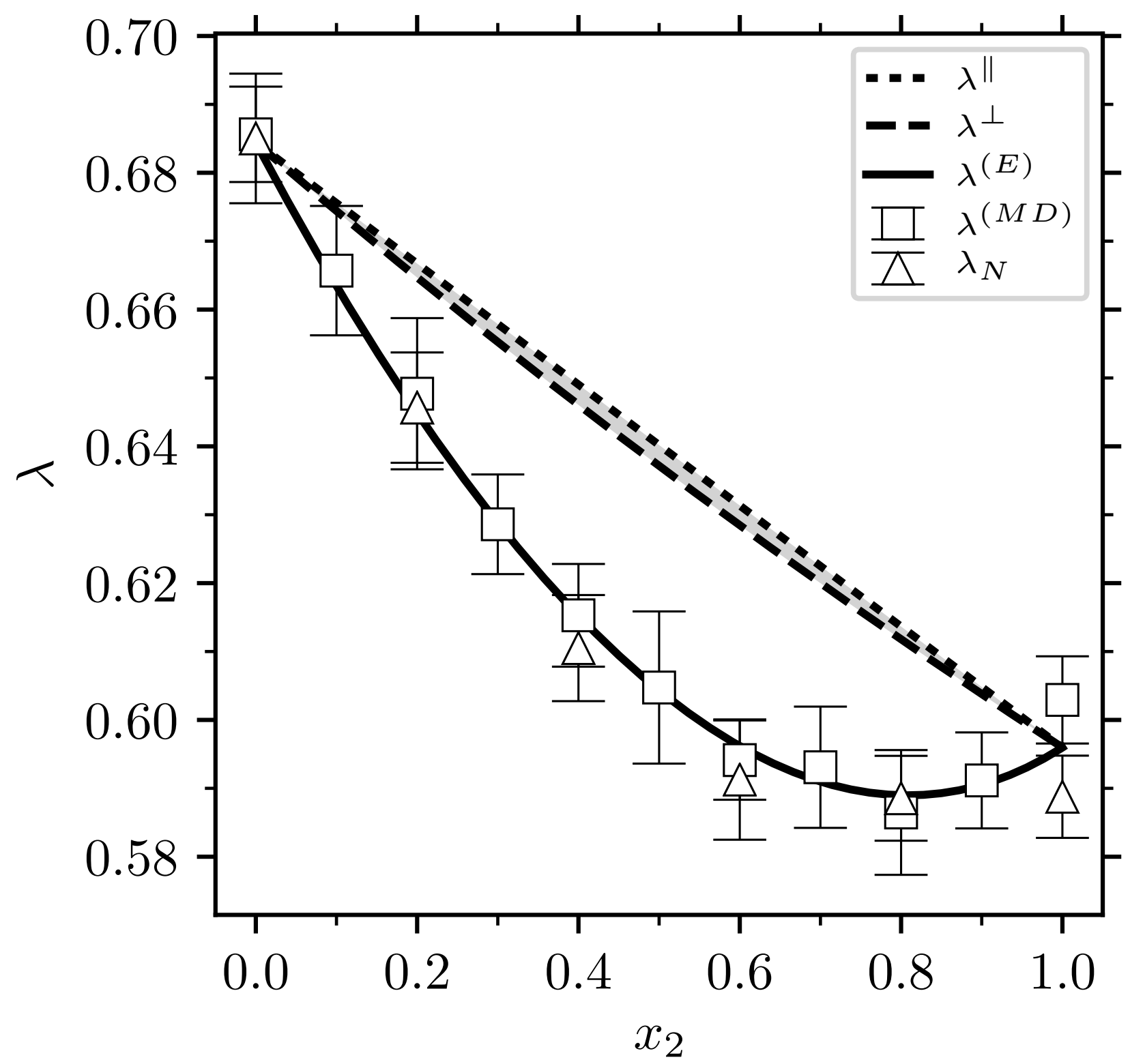

FIG. 4: The extrapolated infinite system-size NEMD, $\lambda_{N}$, (triangles) and equilibrium steadystate, $\lambda^{(M D)}$, (square) thermal conductivities as a function of the non-unit species mole fraction $x_{2}$. Parallel (dotted line) and series (dashed line) limits bound the region of classical/continuum values (shaded). The revised Enskog theory predictions for the steady-state conductivity, $\lambda^{(E)}$, (solid line) are in excellent agreement with the simulation results. A slight apparent overestimation arises from the remaining system-size dependence of our NEMD results. 
skog theory to determine the extent of the anomalous behavior. Fig. 5 maps the maximum possible departure from series or parallel bounds achievable by varying the mole fraction of the system for a particular mass and size ratio. A reduced pressure of $p \sigma_{1}^{3} /\left(k_{B} T\right)=10^{-7}$, corresponding to packing fractions below 0.044 for this parameter space, is used in these calculations to reveal the symmetry of the map in the ideal gas limit which is otherwise prevented by pure species 2 freezing (which occurs at a size ratio of $\sigma_{2} / \sigma_{1} \gtrsim 4.87$ for $p \sigma_{1}^{3} /\left(k_{B} T\right)=0.01$; however, this change makes little difference to the results below this boundary). Anomalous enhancement above the parallel bounds is found at extreme massratios with size-ratios near unity. Reductions below the series bounds are also found for smaller but heavier spheres. Surprisingly, a maximum achievable reduction in thermal conductivity is found which is $\approx 40 \%$ below the series limit (see the cross in Fig. 5). The bulk of the anomalous parameter space lies in the larger-but-lighter region of the map; however, the region of anomalous reduction approaches the line where the species 2 mass scales with its volume which is the experimentally relevant region.

To explore the experimental relevance of the results, the kinetic diameter and molecular mass ratios for combinations of the noble gases, along with nitrogen and hydrogen gas, are plotted in Fig. 6. The map indicates that several real mixtures may exhibit anomalous dehancements; however, care should be taken to verify this as the Enskog theory expressions used here do not take into account the additional degrees of freedom of diatomic gases and in general are not capable of quantitatively predicting the behaviour of real gases, only general trends. It is expected that more complex molecules can exhibit more extreme effective size and mass ratios, although the anomalous enhancement region probably remains inaccessible and the applicability of the hard sphere model is dubious in this limit.

In conclusion, the observed thermal conductivity of binary hard-spheres can exhibit values which lie outside the limits of series-parallel resistance, in agreement with experimental results on $\mathrm{He}-\mathrm{H}_{2}$ systems. These "anomalous" results are present even in the ideal gas limit, which implies that they cannot be explained by any structural/clustering effect such as those which are prevalent in the nanofluid literature. To better understand results in nanofluids, liquid densities can be explored using the techniques outlined here; however, the binary hardsphere model has two serious shortcomings: an ideal-gas heat capacity and the absence of a gas-liquid transition. Future work will explore adding internal degrees of freedom to the spheres to account for varying heat capacity which will allow a better parameterization of real 


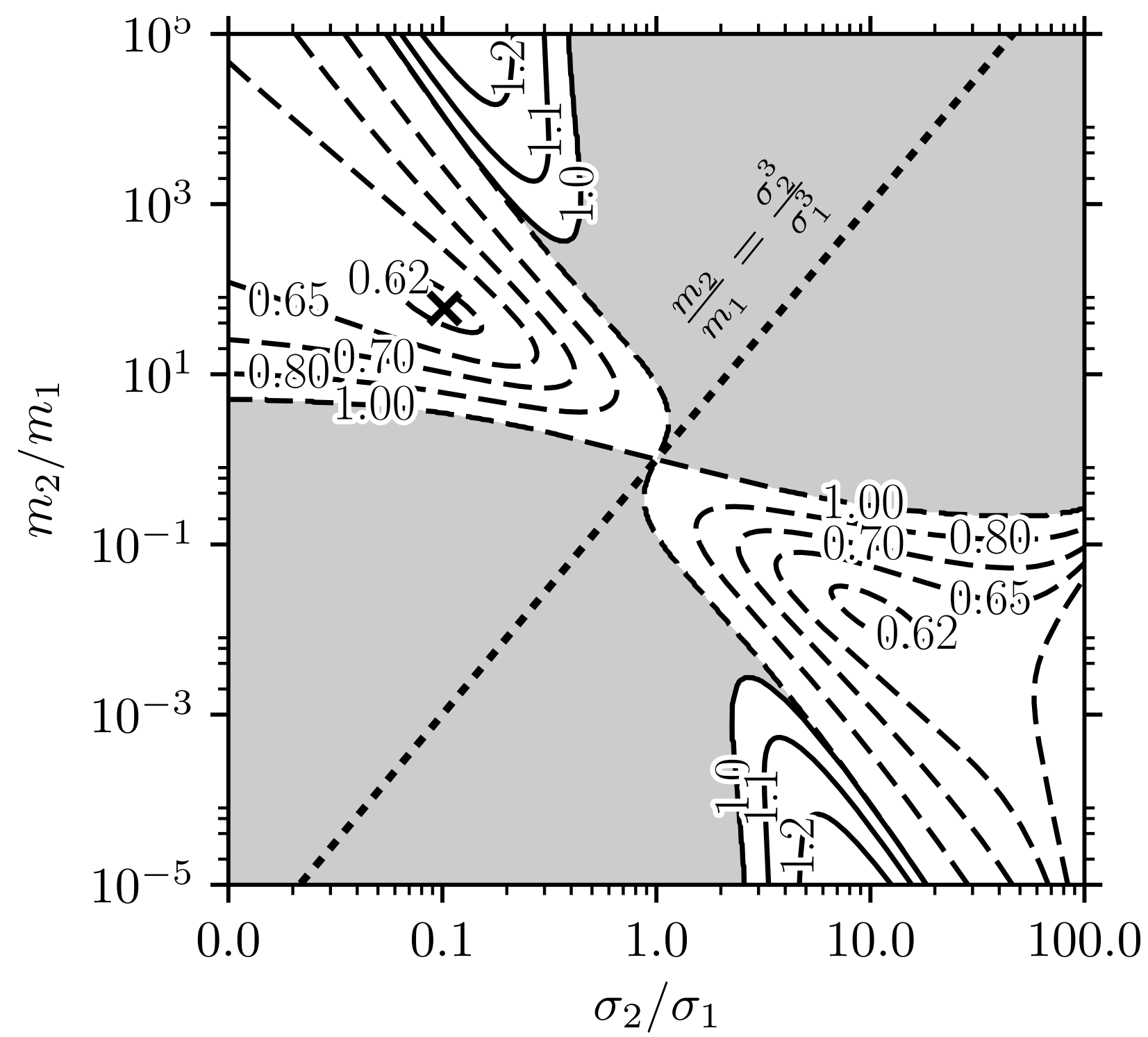

FIG. 5: A contour map of steady-state thermal conductivities outside the series (dashed) and parallel (solid) bounds as a function of mass and size ratio. The contours denote the increase/decrease of the thermal conductivity relative to the parallel/series bound at the concentration of maximum deviation. A cross indicates the maximum decrease of the steady-state thermal conductivity which occurs at $m_{2} / m_{1} \approx 59.6$ and $\sigma_{2} / \sigma_{1} \approx 0.102$. A dotted line indicates where mass scales with molecular volume, assuming a constant density. 


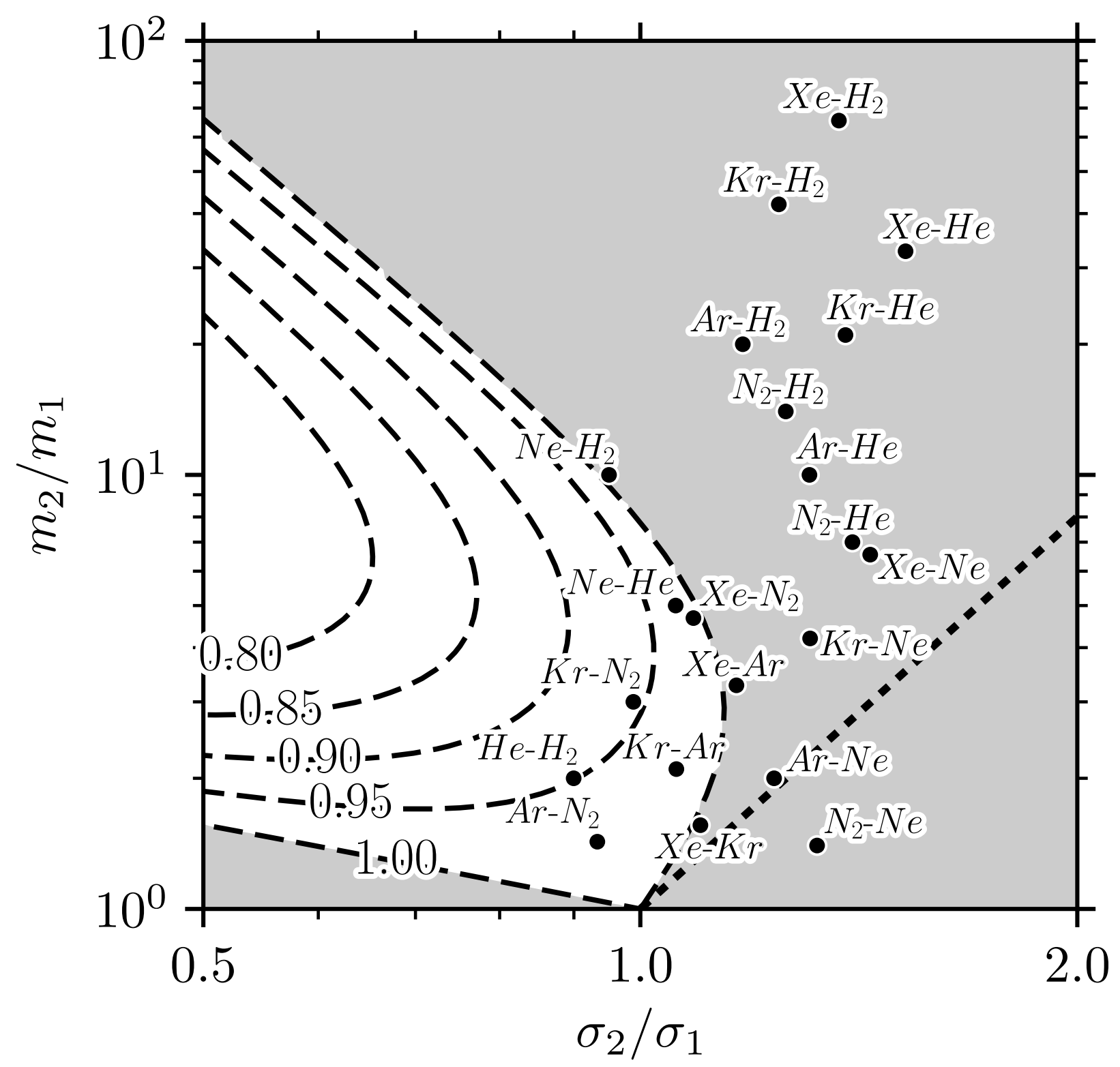

FIG. 6: As described in Fig. 5 but focused on the parameter space relevant for the noble gases, hydrogen, and nitrogen. Kinetic diameters are taken from Ref. [26-28]

fluids. Attractive systems, such as square-wells, may also be used to explore liquid systems which do not have strong density-pressure dependences, and the comparison at pressures where the nanoparticle forms a crystalline phase will be explored. Finally, work on a multiscale modelling approach using kinetic theory to provide phenomenological closures to a hydrodynamic description is underway to allow fluid dynamics simulations of nanofluidic devices where transient effects may dominate over steady-state conduction. 


\section{Acknowledgements}

The authors acknowledge the support of the Maxwell computing service at University of Aberdeen, and the Aberdeen-Curtin Alliance [30] between the University of Aberdeen (Scotland, UK) and Curtin University (Perth, Australia) which is funding the PhD of Craig Moir. Paolo Raiteri and Julian Gale thank the Australian Research Council for funding.

* Electronic address: m.campbellbannerman@abdn.ac.uk

[1] J. A. Eastman, S. U. S. Choi, S. Li, W. Yu, and L. J. Thompson, Appl. Phys. Lett. 78, 718 (2001).

[2] P. Keblinski, R. Prasher, and J. Eapen, J. Nanopart. Res. 10, 1089 (2008).

[3] J. Buongiorno, D. C. Venerus, N. Prabhat, T. McKrell, J. Townsend, R. Christianson, Y. V. Tolmachev, P. Keblinski, L.-w. Hu, J. L. Alvarado, et al., J. Appl. Phys. 106, 094312 (2009).

[4] J. Eapen, R. Rusconi, R. Piazza, and S. Yip, J. Heat Transfer 132, 102402 (2010).

[5] Y. Hwang, J. K. Lee, C. H. Lee, Y. M. Jung, S. I. Cheong, C. G. Lee, B. C. Ku, and S. P. Jang, Thermochim. Acta 455, 70 (2007).

[6] S. de Groot and P. Mazur, Non-Equilibrium Thermodynamics (Dover, New York, 1984).

[7] J. J. Erpenbeck, Phys. Rev. A 39, 4718 (1989).

[8] S. Bastea, Phys. Rev. E 75, 031201 (2007).

[9] J. Armstrong and F. Bresme, Phys. Chem. Chem. Phys. 16, 12307 (2014).

[10] J. J. Erpenbeck, Phys. Rev. A 45, 2298 (1992).

[11] J. J. Erpenbeck, Phys. Rev. E 48, 223 (1993).

[12] D. M. Heyes, J. Chem. Phys. 96, 2217 (1992).

[13] J. Eapen, J. Li, and S. Yip, Phys. Rev. Lett. 98, 028302 (2007).

[14] N. A. T. Miller, P. J. Daivis, I. K. Snook, and B. D. Todd, J. Chem. Phys. 139, 144504 (2013).

[15] M. N. Bannerman and L. Lue, J. Chem. Phys. 130, 164507 (2009).

[16] M. N. Bannerman, R. Sargant, and L. Lue, J. Comput. Chem. 32, 3329 (2011).

[17] M. López de Haro, E. G. D. Cohen, and J. M. Kincaid, J. Chem. Phys. 78, 2746 (1983).

[18] M. J. Lindenfield and B. Shizgal, Chem. Phys. 41, 81 (1979).

[19] P. Mukhopadhyay and A. K. Barua, J. Appl. Phys. 18, 635 (1967). 
[20] L. Biolsi and E. A. Mason, J. Chem. Phys. 54, 3020 (1971).

[21] A. G. Shashkov, F. P. Kamchatov, and T. N. Abramenko, J. Eng. Phys. 24, 461 (1973).

[22] T. Pöschel and T. Schwager, Computational Granular Dynamics (Springer, New York, 2005).

[23] B. D. Freeman, Macromolecules 32, 375 (1999).

[24] M. S. Green, J. Chem. Phys. 22, 398 (1954).

[25] R. Kubo, J. Phys. Soc. Jpn. 12, 570 (1957).

[26] D. W. Breck, Zeolite Molecular Sieves: Structure, Chemistry, and Use (Wiley, New York, 1973).

[27] A. F. Ismail, K. Khulbe, and T. Matsuura, Gas Separation Membranes (Springer, New York, 2015).

[28] S. Matteucci, Y. Yampolskii, B. D. Freeman, and I. Pinnau, Materials Science of Membranes for Gas and Vapor Separation (Wiley, 2006), chap. Transport of Gases and Vapors in Glassy and Rubbery Polymers.

[29] It should be noted that this is not the only definition of the thermal conductivity which is independent of the choice of fluxes. For example, another definition can be derived by assuming no concentration gradients are present (e.g., at the onset of thermal conduction) but allowing chemical potential gradients to arise due to gradients in the temperature. This is not explored here as it is demonstrated that $\lambda_{N} \approx \lambda$ at steady state, but this definition may have relevance for systems undergoing rapid changes in temperature.

[30] http://aberdeencurtinalliance.org 